\title{
Control of electrical resistance and magnetoresistance by electric-field-driven oxygen ion migration in a single $\mathrm{GdO}_{x}$ wire
}

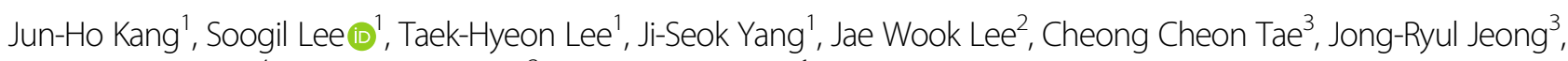
Seung-Young Park ${ }^{4}$, Byong-Guk Park $\mathbb{B}^{2}$ and Kab-Jin Kim (1)

\begin{abstract}
Electric-field-driven ion migration can significantly modulate the electric and magnetic properties of solids, creating novel functionalities useful for advanced electromagnetic devices. Earlier works have used vertically stacked structures for this purpose, in which the redox process results from ion migration driven by a vertical electric field through the interfaces. However, the existence of the interfaces between the dissimilar layers causes the oxidation and reduction processes to have high and asymmetric energy barriers, which means that a large electric field is required to control the devices. Here, we show that in a partially oxidized single $\mathrm{GdO}_{x}$ wire using a lateral electric field configuration, low and symmetric energy barriers for the oxidation and reduction processes can be achieved. We provide evidence that the redox process is the result of the lateral motion of oxygen ions by directly visualizing the electric-field-driven realtime ionic motion using an optical microscope. An electric field as low as $10^{5} \mathrm{~V} / \mathrm{m}$ was able to drive oxygen ions at room temperature, allowing controllable modulation of the electrical resistance using a lateral electric field. A large negative magnetoresistance was also observed in the $\mathrm{GdO}_{x}$ wire, and its magnitude was significantly enhanced up to $20 \%$ at $9 \mathrm{~T}$ through oxygen ion control. Our results suggest that the electrical and magnetic properties of single $\mathrm{GdO}_{x}$ can be efficiently controlled through oxygen ion motion driven by a lateral electric field, which paves the way for fully functional electromagnetic devices such as artificial synapses.
\end{abstract}

\section{Introduction}

Gadolinium (Gd) is one of the most interesting rareearth metals. It exhibits fascinating electrical and magnetic properties ${ }^{1-4}$ and has practical value in applications including hydrogen storage ${ }^{5}$ and CMOS technology as gate electrodes $^{6}$ and spintronics ${ }^{7-12}$. One distinctive property of $\mathrm{Gd}$ is that it is very easily oxidized, forming oxide compounds, $\mathrm{GdO}_{x}$. Since the oxygen ions are mobile in $\mathrm{GdO}_{x}{ }^{13,14}$, it has been used as an oxygen source (or sink) in ferromagnet/ $\mathrm{GdO}_{x}$ heterostructures ${ }^{14-19}$. In these heterostructures, the electric and magnetic

\footnotetext{
Correspondence: Soogil Lee (lee.soogil.83@kaist.ac.kr) or

Kab-Jin Kim (kabjin@kaist.ac.kr)

'Department of Physics, KAIST, Daejeon 34141, Korea

${ }^{2}$ Department of Materials Science and Engineering, KAIST, Daejeon 34141, Korea

Full list of author information is available at the end of the article
}

properties of the ferromagnet can be modulated by the redox process through electric-field-driven $\mathrm{O}^{2-}$ migration between the ferromagnet and $\mathrm{GdO}_{x}$. However, the vertical oxygen migration in the heterostructures has an inherent asymmetry, which leads to different energy barriers for oxidation and reduction ${ }^{18}$. Furthermore, the energy barrier for oxygen migration is generally large, so the use of a high electric field is required to control the device ${ }^{16,17}$. Therefore, for $\mathrm{GdO}_{x}$ to be properly utilized in practical devices, it is highly necessary to achieve a symmetric and low energy barrier for oxygen ion motion.

In this work, we investigate the possibility of utilizing oxygen ions in a single $\mathrm{GdO}_{x}$ device instead of heterostructures. Contrary to previous works ${ }^{14-19}$, we explore the lateral motion of oxygen ions by applying a lateral electric field. By employing optical and electrical 
measurements, we find that the oxygen ions are transported along the $\mathrm{GdO}_{x}$ layer in a controllable way with almost the same energy barriers for oxidation and reduction. Furthermore, the energy barrier is found to be much smaller than in previous reports, which we ascribe to the low concentration of oxygen ions in our $\mathrm{GdO}_{x}$. The possibility of practical devices is presented by showing the modulation of the electrical resistance and magnetoresistance (MR) through oxygen ion control in a single $\mathrm{GdO}_{x}$ device.

\section{Materials and methods}

\section{Sample preparation}

For this study, a 10-nm-thick Gd film was deposited on a $\mathrm{Si} / \mathrm{SiN}_{x}(200 \mathrm{~nm})$ substrate by direct current (DC) magnetron sputtering under a working pressure of 3 mTorr. To investigate the oxygen ion migration effect, we used three different capping layers, as shown in Fig. 1a. The first sample had 3-nm-thick $\mathrm{SiN}_{x}$ as a capping layer. For this sample, we intentionally increased the radio frequency (RF) sputtering power up to $150 \mathrm{~W}$ to create a bumpy surface. This allowed oxygen to penetrate into the Gd layer and form $\mathrm{GdO}_{x}$ when the sample was exposed to air. The second sample was capped by a 1.5$\mathrm{nm}$-thick Ta layer using DC magnetron sputtering. When the sample was exposed to air, the Ta oxidized, and the oxygen ions in $\mathrm{TaO}_{x}$ diffused into the Gd layer and formed $\mathrm{GdO}_{x}$. The third sample was prepared as a control sample with a much thicker $\operatorname{SiN}_{x}$ capping layer. For this, 20-nm-thick $\mathrm{SiN}_{x}$ was deposited by RF magnetron sputtering. To make a smooth surface, we reduced the RF sputtering power to as low as $50 \mathrm{~W}$. We expected that in this case, Gd would not be oxidized at all and would remain in a metallic state (see Supplementary Information 1 for more details). For the electrical measurements, the films were patterned into microwires with a Hall-bar structure by conventional photolithography and Ar-ion milling techniques, as shown in Fig. 1b. The wire lengths were set to $55 \mu \mathrm{m}$ with varying widths of 5,10 , and $20 \mu \mathrm{m}$. We used the four-probe measurement technique, which separates the contacts for the input current and output voltage (see Fig. 1b).

\section{Results and discussion}

\section{Electrical and optical measurements of ion migration}

Figure $1 \mathrm{c}-\mathrm{e}$ shows the temporal variation in the longitudinal resistivity $\left(\rho_{x x}\right)$ of the three different samples. To monitor the differences in resistance, we fixed the current, whose magnitude corresponded to an electric field of $10^{4}-10^{5} \mathrm{~V} / \mathrm{m}$, depending on the resistance. The resistivity of partially oxidized $\mathrm{Gd}$, that is, $\mathrm{SiN}_{x} / \mathrm{GdO}_{x}$ and $\mathrm{TaO}_{x} /$ $\mathrm{GdO}_{x}$ (Fig. 1c, d), decreased drastically over time, while that of metallic Gd was almost constant over time (Fig. 1e).
This large decrease in resistivity indicates that a reduction process, from insulator-like $\mathrm{GdO}_{x}$ to metal-like $\mathrm{Gd}$, occurs when the oxygen ions are driven by an electric field. To provide more direct evidence, we measured the real-time color change of $\mathrm{TaO}_{x} / \mathrm{GdO}_{x}$ using an optical microscope because the change in oxygen ion concentration is reflected by changes in the optical contrast ${ }^{20}$. We first applied a negative electric field for $1000 \mathrm{~s}$ to push the oxygen ions to the right edge of the wire and then reversed the electric field polarity. Figure if shows optical microscope images taken after reversing the polarity of the electric field. We set the initial image $(t=0)$ as a background image and subtracted it from all other images taken at $t>0$. The black region indicates a decrease in oxygen ions, while the white region represents an increase in oxygen ions. The result shows that the accumulated oxygen ions move towards the anode under the electric field.

Figure $1 \mathrm{~g}$ shows the variations in the resistances of electrodes $\mathrm{A}-\mathrm{B}$ and electrodes $\mathrm{B}-\mathrm{C}$, which were measured at the same time as the optical images. The results show that there is a time delay in the resistance variation between the physically separated regions, which suggests that the variation in resistivity is caused by the oxygen ion migration driven by the electric field. To further confirm the oxygen migration, we measured the time delay by changing the electric field. Figure $1 \mathrm{~h}$ clearly shows that the time delay decreases with increasing electric field, which is consistent with the oxygen migration model. We observed similar oxygen ion motion in $\mathrm{SiN}_{x} / \mathrm{GdO}_{x}$, but for metallic $\mathrm{Gd}$, we did not observe any color change (Supplementary Information 2). All these observations suggest that the variation in resistivity observed in $\mathrm{GdO}_{x}$ originates from the electric-field-driven oxidation and reduction processes induced by lateral oxygen ion migration (further discussions are presented in Supplementary Information 3 and 4).

\section{Energy barriers for ion migration}

We next studied the oxygen ion migration in a more quantitative way. Figure $2 \mathrm{a}$ shows the time-dependent resistance variation in $\mathrm{GdO}_{x}$ at temperatures from $75^{\circ} \mathrm{C}$ to $90^{\circ} \mathrm{C}$ at a $5^{\circ} \mathrm{C}$ interval. Here, the temperatures were set higher than room temperature to obtain a fast resistance change (i.e., rapid oxygen ion migration). We applied an electric field of $1 \times 10^{4} \mathrm{~V} / \mathrm{m}$. As we increase the temperature, the slope of the resistance variation increases for both the oxidation and reduction processes. This implies that the ionic motion occurs through a thermal activation process. To quantitatively determine the activation energy barrier, we plot the logarithm of the resistance variation rate $\left(\Delta R_{x x} / \Delta t\right)$ with respect to the inverse of temperature in Fig. 2b. The linear dependence in Fig. 2b suggests that the ionic motion follows the Arrhenius law, which we can use to estimate the activation energy barrier. 
(a)

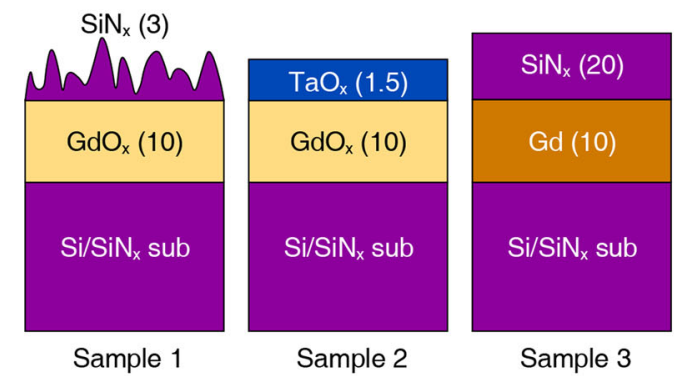

(c)

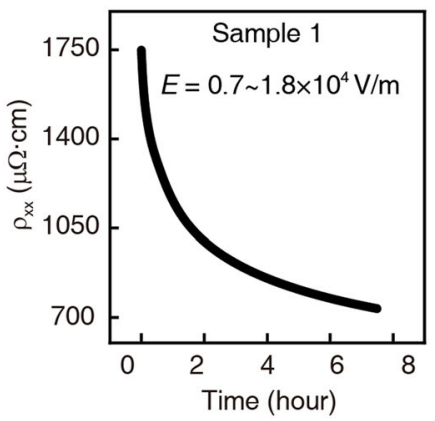

(f)
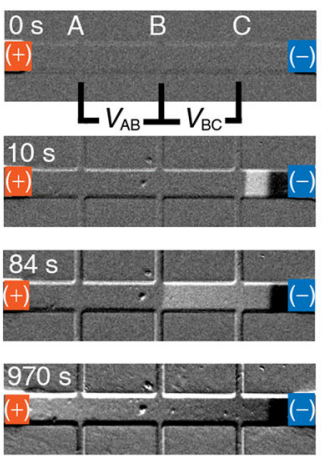

(d)

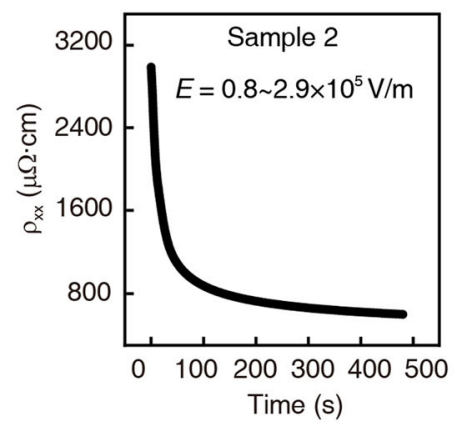

(g)

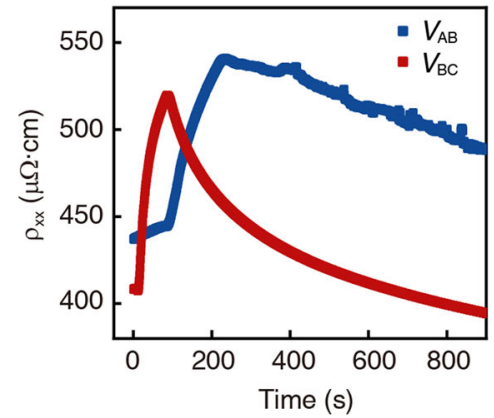

(b)

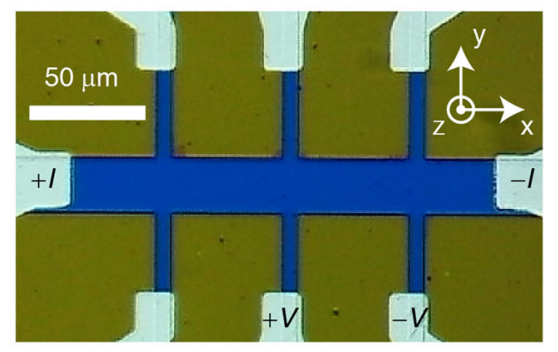

(e)

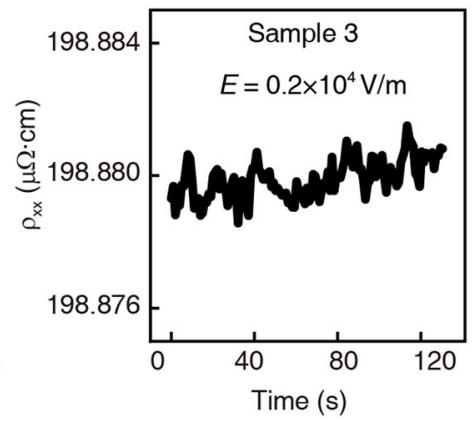

(h)

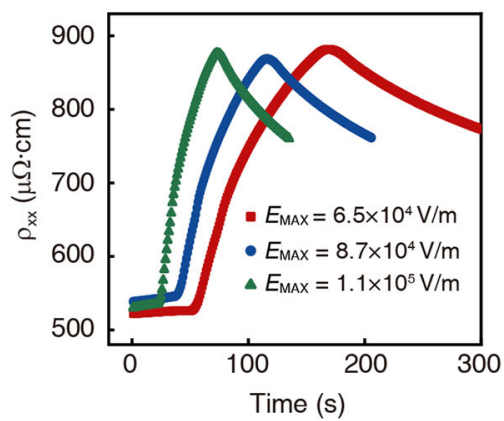

Fig. 1 Electrical and optical measurements of oxygen ion migration in $\mathbf{G d O}_{\mathbf{x}}$ wire. a Schematic illustration of the three types of Gd film. b Microscope image of a patterned device. The four-probe geometry used for electrical measurements is marked on the image. c Time-dependent resistivity of the $\mathrm{SiN}_{x}(3 \mathrm{~nm}) / \mathrm{GdO}_{x}(10 \mathrm{~nm})$ device for $j=1 \times 10^{5} \mathrm{~A} / \mathrm{cm}^{2}\left(E=0.7-1.8 \times 10^{4} \mathrm{~V} / \mathrm{m}\right)$ at $300 \mathrm{~K}$. d Time-dependent resistivity of the TaO $\mathrm{O}_{x}(1.5 \mathrm{~nm}) /$ $\mathrm{GdO}_{x}(10 \mathrm{~nm})$ device for $j=1 \times 10^{6} \mathrm{~A} / \mathrm{cm}^{2}\left(E=0.8-2.9 \times 10^{5} \mathrm{~V} / \mathrm{m}\right)$ at $300 \mathrm{~K}$. e Time-dependent resistivity of the $\mathrm{SiN}_{x}(20 \mathrm{~nm}) / \mathrm{Gd}(10 \mathrm{~nm})$ device for $j=1 \times 10^{5} \mathrm{~A} / \mathrm{cm}^{2}\left(E=0.2 \times 10^{4} \mathrm{~V} / \mathrm{m}\right)$ at $300 \mathrm{~K}$. $\mathbf{f}$ Real-time images of the migration of accumulated oxygen ions in the $\mathrm{TaO}_{x} / \mathrm{GdO}_{x}$ device for $j=2 \times 10^{6} \mathrm{~A} / \mathrm{cm}^{2}$. g Resistivity changes observed as accumulated oxygen ions passed though the detecting electrodes. $\mathbf{h}$ Resistivity modulation by oxygen ion migration depends on the amplitude of the electric field.

The obtained energy barriers were found to be $0.49 \pm$ 0.04 and $0.48 \pm 0.01 \mathrm{eV}$ for the oxidation and reduction processes, respectively. These values are much smaller than the binding energy of $\mathrm{Gd}-\mathrm{O}$ in gadolinium oxide $\left(\mathrm{Gd}_{2} \mathrm{O}_{3}\right)$, but comparable to a previously reported oxygen ion diffusion energy barrier ${ }^{13}$. This suggests that the motion of oxygen ions in $\mathrm{GdO}_{x}$ occurs via successive jumps from $\mathrm{Gd}_{2} \mathrm{O}_{3}$ to $\mathrm{Gd}$. In this process, the energy used to destroy the old bond and that used to create the new bond are almost the same, so the energy barrier to movement of the oxygen ions can be small ${ }^{14}$. It should be noted that the energy barriers obtained here are different from those in vertically stacked heterostructures. In heterostructures, the energy barriers are higher than ours and are asymmetric for oxidation and reduction (the energy barrier for the reduction process is higher than that for the oxidation process $)^{18}$. Considering that the diffusion energy barrier depends on the oxygen ion concentration $^{21}$, the smaller energy barrier in our device is possibly due to the low oxygen concentration of our 


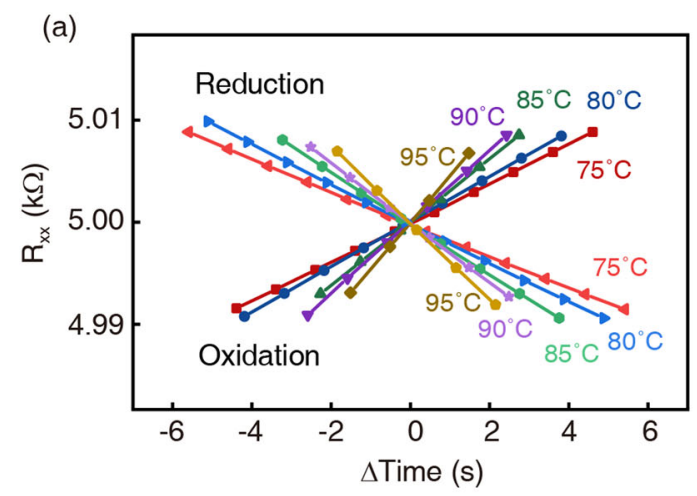

(b)
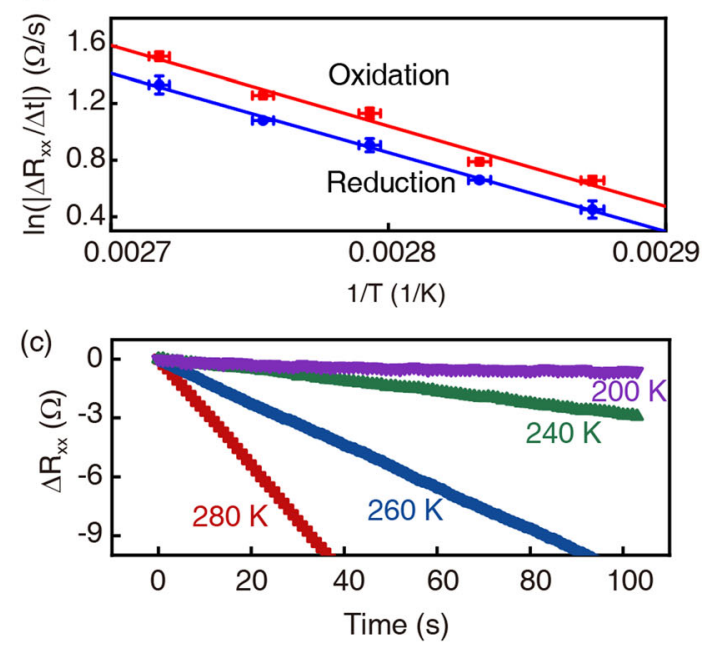

Fig. 2 Determination of energy barriers for ion migration. a Temporal dependence of longitudinal resistance $R_{x x}$ at various temperatures in the $\mathrm{SiN}_{x}(3 \mathrm{~nm}) / \mathrm{GdO}_{x}(10 \mathrm{~nm})$ device. Here, $R_{x x}$ was measured by repeatedly switching the electric field polarity at the lower bound $\left(R_{x x}^{\text {low }}=4.99 \mathrm{k} \Omega\right)$ and upper bound $\left(R_{x x}^{\text {high }}=5.01 \mathrm{k} \Omega\right)$, as shown in Fig. 3. An electric field of $1 \times 10^{4} \mathrm{~V} / \mathrm{m}$ was applied. A positive slope indicates the oxidation process, and a negative slope corresponds to the reduction process. Here, $\Delta$ Time=Time-Time at $5.00 \mathrm{k} \Omega$. b Arrhenius plot obtained from $\mathbf{a}$. The detailed fitting function is discussed in Supplementary Information 5 and 6. c Variation in $R_{x x}$ produced by the reduction process in the $\mathrm{GdO}_{x}$ device at various temperatures. Here, all the measurements were performed in a vacuum chamber under a $10 \mathrm{mbar}$ pressure and $0.7 \%$ humidity.

$\mathrm{GdO}_{x}$, which can be supported by the low resistivity of our $\mathrm{GdO}_{x}\left(\sim 10^{3} \mu \Omega \cdot \mathrm{cm}\right)$ compared to that of $\mathrm{GdO}_{x}$ in previous works $\left(\sim 10^{11} \mu \Omega \cdot \mathrm{cm}\right)^{18}$. The asymmetry in the energy barrier in the heterostructures arises because the oxidation and reduction occur as a result of oxygen ion motion across the interfaces between dissimilar layers. In our case, on the other hand, oxidation and reduction occur in a single $\mathrm{GdO}_{x}$ wire layer through the lateral motion of oxygen ions, resulting in similar energy barriers for oxidation and reduction.

It should also be noted that despite having similar energy barriers, the oxidation and reduction processes are not exactly equivalent in our $\mathrm{GdO}_{x}$ wires. This can be inferred from the slight offset shown in Fig. 2b. The offset indicates that the reduction process is slower than the oxidation process due to the concentration gradient of oxygen ions ${ }^{22}$. In the reduction process, the distributed oxygen ions are driven by an electric field and accumulate at the anode. In the oxidation process, on the other hand, the accumulated oxygen ions are spread out over the wire. As a result, the concentration-gradient-induced ion diffusion is additive to the electric-field-induced ion migration for the oxidation process, while it is subtractive for the reduction process. This generates the slightly different resistance variation rates for oxidation and reduction (for a more quantitative analysis, see Supplementary Information 5 and 6).

Since the oxygen ions are driven by a thermal activation process with finite energy barriers, ionic motion can be generally suppressed at low temperature. Figure 2c shows the time-dependent resistance variation in the $\mathrm{GdO}_{x}$ wire at various temperatures. The results show that the resistance variation is gradually suppressed with decreasing temperature, and the resistance becomes stable below $200 \mathrm{~K}$, suggesting that the thermal activation of oxygen ions is suppressed below $200 \mathrm{~K}$.

\section{Mimicking the synaptic function using ion migration}

The electric-field-driven oxygen ion migration and resulting resistance variation enable control of the device resistance in a more active way. To verify this, we repeatedly switched the electric field polarity at the lower bound $\left(R_{x x}^{\text {low }}=3.00 \mathrm{k} \Omega\right)$ and upper bound $\left(R_{x x}^{\text {high }}=\right.$ $3.06 \mathrm{k} \Omega$ ) as shown in Fig. 3a. The device resistance periodically changes between the two resistance states, indicating that oxygen ions move back and forth within the wire. This controllable quasilinear variation in device resistance can be used in artificial synaptic devices ${ }^{22}$. Figure $3 \mathrm{a}-\mathrm{c}$ shows the variation in resistance for various electric fields $\left(0.55-1.64 \times 10^{5} \mathrm{~V} / \mathrm{m}\right)$. The higher-amplitude and longer-duration electric fields lead to a larger change in resistance. If we treat the device resistance as a synaptic weight, then this phenomenon closely mimics the transmission characteristics of a biological synapse. Furthermore, the sign of the resistance variation can also be controlled by the electric field polarity, which can be used to mimic the potentiation and depression functions of a biological synapse.

\section{Effect of ion migration on the MR}

We finally investigated the effect of oxygen ions on the MR of $\mathrm{GdO}_{x}$. To investigate the MR of $\mathrm{GdO}_{x}$, we first controlled the device resistivity by applying an electric field at room temperature, as shown in Fig. 1c, d, and then cooled it down to a low temperature to stabilize the 


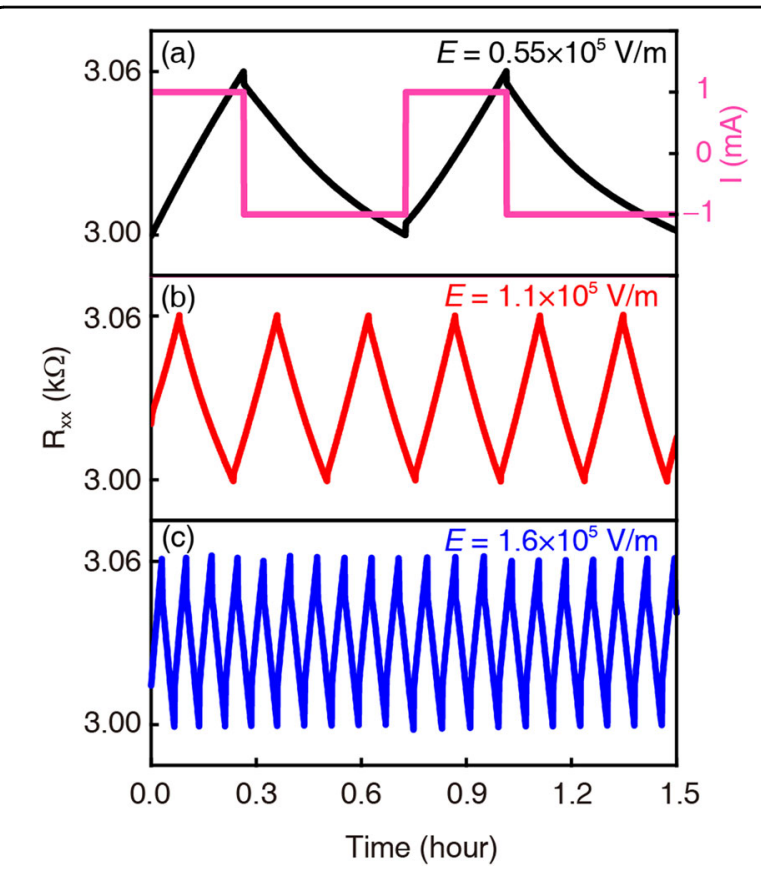

Fig. 3 Mimicking the synaptic function using ion migration. Longitudinal resistance $\left(R_{x x}\right)$ with repeated switching of the current (I) direction under a current of $\mathbf{a} 1 \mathrm{~mA}\left(E=0.55 \times 10^{5} \mathrm{~V} / \mathrm{m}\right), \mathbf{b} 2 \mathrm{~mA}$ $\left(E=1.1 \times 10^{5} \mathrm{~V} / \mathrm{m}\right)$, or $\mathbf{c} 3 \mathrm{~mA}\left(E=1.6 \times 10^{5} \mathrm{~V} / \mathrm{m}\right)$ in the $\mathrm{SiN}_{x}(3 \mathrm{~nm}) / \mathrm{GdO}_{x}$ $(10 \mathrm{~nm})$ device. When $R_{x x}<R_{\text {LOWER }}=3.00 \mathrm{k} \Omega$ or $R_{x x}>R_{H I G H}=3.06 \mathrm{k} \Omega$ was met, the current direction (electric field polarity) was switched.

resistance. By applying an appropriate electric field at room temperature, we achieved two different oxygen ion concentration states with resistivity, $\rho_{x x}$, values of 2200 and $1700 \mu \Omega \cdot \mathrm{cm}$. Figure $4 \mathrm{a}$ shows the temperature-dependent resistivity for two oxygen-concentration-controlled samples (red and blue symbols) and metallic Gd (green symbol). The temperature coefficient of resistivity, $\frac{\mathrm{d} \rho_{x x}}{\mathrm{~d} T}$, was found to be positive for metallic Gd (green symbols), while it was negative for the oxygen-concentration-controlled samples (red and blue symbols). This indicates that oxygen ion inclusions make the system exhibit a more insulator-like state.

The MR was then measured at $10 \mathrm{~K}$, which is far lower than the Curie temperature of $\mathrm{GdO}_{x}$ (for a discussion about the Curie temperature, see Supplementary Information 7). Figure $4 \mathrm{~b}$ shows that all three samples exhibit a negative MR, that is, the resistance decreases as the magnetic field increases ${ }^{23}$. In metallic ferromagnets, a negative $M R$ is usually attributed to suppression of magnons (or spin fluctuations) by an external magnetic field $^{23-25}$. However, here, we found that the slope of the negative MR could be remarkably enhanced by increasing the oxygen ion concentration. The variation in MR reached $20 \%$ at $9 \mathrm{~T}$ for the $\rho_{x x}=2200 \mu \Omega \cdot \mathrm{cm}$ sample.

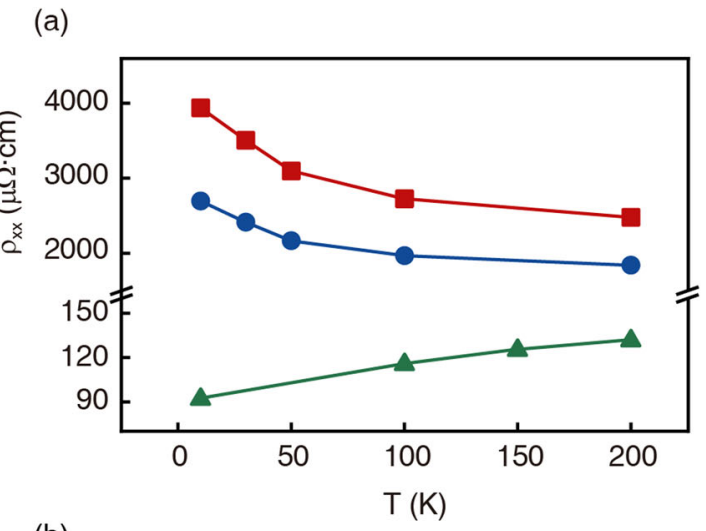

(b)
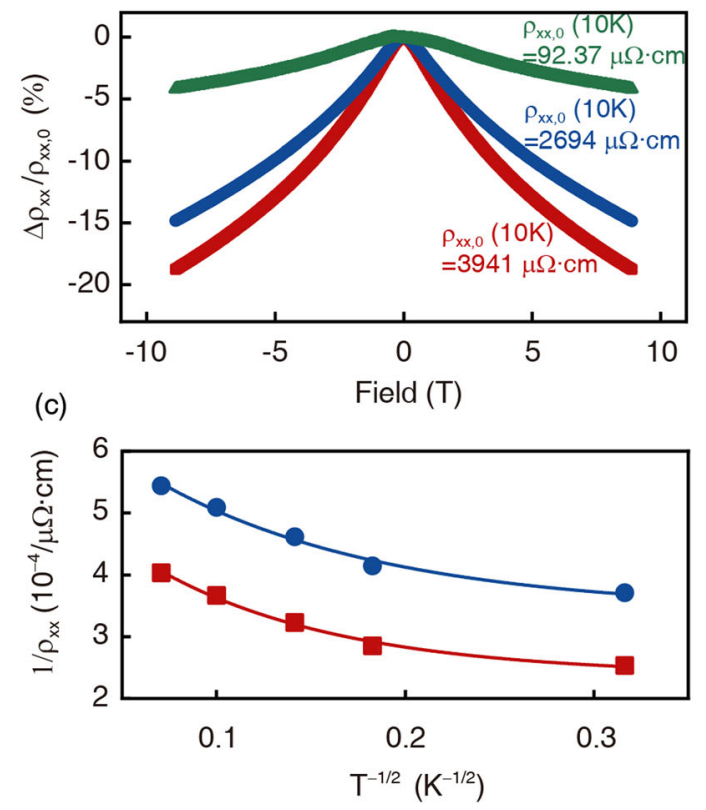

Fig. 4 Effect of ion migration on the magnetoresistance. a Temperature-dependent resistivity for oxygen-concentrationcontrolled $\mathrm{GdO}_{x}$ (red and blue symbols) and metallic $\mathrm{Gd}$ (green symbol). $\mathbf{b}$ Magnetic-field-dependent resistivity of the three samples at $10 \mathrm{~K}$. The colors correspond to those in $\mathbf{a}$. c Relationship between $1 / \rho_{x x}$ and $T^{-1 / 2}$ (the data are replotted from $\mathbf{a}$ ). The solid lines represent fitting curves based on Eq. (1).

The large enhancement in MR observed in Fig. 4b can be explained by the tunneling giant MR (tunneling GMR) that generally appears in inhomogeneous granular films ${ }^{26-28}$. In magnetically inhomogeneous media with nonaligned ferromagnetic entities, it has been found that the resistivity can be gradually decreased by applying a magnetic field since the magnetic field aligns the ferromagnetic entities parallel to each other. This leads to a low resistance state in tunneling transport.

The tunneling model can be further supported by percolation theory. According to percolation theory, metallic conduction and hopping (or tunneling) conduction 
coexist in partially oxidized systems. Then, the temperature dependence of the resistivity can be given by ${ }^{28-30}$

$$
\frac{1}{\rho_{x x}}=\frac{1}{\rho_{\mathrm{t}}} \exp \left(-2 \sqrt{\frac{c}{k T}}\right)+\frac{1}{\rho_{\mathrm{c}}},
$$

where $c$ is the activation energy for electron tunneling, $\rho_{\mathrm{t}}$ is the resistivity of the tunneling channel, and $\rho_{\mathrm{c}}$ is the resistivity of the metallic channel. Figure 4c shows $\rho_{x x}^{-1}$ versus $T^{-1 / 2}$ for two insulator-like samples (replotted from Fig. 4a). The curve shows the best fitting based on Eq. (1). The results indicate that $\rho_{\mathrm{t}}=2900 \mu \Omega \cdot \mathrm{cm}$ and $\rho_{\mathrm{c}}=4200 \mu \Omega \cdot \mathrm{cm}$ for the red curve and that $\rho_{\mathrm{t}}=$ $2700 \mu \Omega \cdot \mathrm{cm}$ and $\rho_{\mathrm{c}}=2900 \mu \Omega \cdot \mathrm{cm}$ for the blue curve. This implies that the contribution of tunneling conduction is comparable to or even more dominant than that of metallic conduction in our $\mathrm{GdO}_{x}$, which verifies the tunneling-induced MR. Last, we would like to emphasize that unlike previous reports where the tunneling GMR was controlled by the material composition ${ }^{26-28}$, in our $\mathrm{GdO}_{x}$ device, the MR can be easily controlled by tuning the oxygen ion migration using an electric field.

\section{Conclusions}

In summary, we investigated lateral-electric-field-driven oxygen ion migration in a partially oxidized Gd device. Using an optical microscope, we directly observed the directional motion of oxygen ions driven by an electric field. The resistance was found to be closely linked to the oxygen ion concentration, allowing the electrical resistance to be greatly modulated by an electric field. We also investigated the magnetic properties of Gd and found that oxygen ions greatly enhanced the magnetoresistance of Gd. Our results therefore suggest that the electrical resistance and magnetoresistance of $\mathrm{Gd}$ can be significantly modulated by electric-field-driven oxygen ion migration, which may enable functional electromagnetic devices such as artificial synapses.

\section{Acknowledgements}

This work was supported by National Research Foundation of Korea (NRF) grants funded by the Korea Government (MSIP) (Grant Nos. 2016R1A5A1008184, 2017R1A2A2A05069760, 2019M3F3A1A02072477, and 2019R1H1A2079946). S.-Y.P. was supported by the National Research Council of Science and Technology (NST) (Grant No. CAP-16-01-KIST) by the Korea government (MSIP).

\section{Author details \\ ${ }^{1}$ Department of Physics, KAIST, Daejeon 34141, Korea. ${ }^{2}$ Department of Materials Science and Engineering, KAIST, Daejeon 34141, Korea. ${ }^{3}$ Department of Materials Science and Engineering, Graduate School of Energy Science and Technology, Chungnam National University, Daejeon 34134, South Korea. ${ }^{4}$ Center for Scientific Instrumentation, KBSI, Daejeon 34133, South Korea}

Conflict of interest

The authors declare that they have no conflict of interest.

\section{Publisher's note}

Springer Nature remains neutral with regard to jurisdictional claims in published maps and institutional affiliations.

Supplementary information is available for this paper at https://doi.org/ 10.1038/s41427-020-0222-y.

Received: 12 August 2019 Revised: 18 March 2020 Accepted: 24 March 2020.

Published online: 19 June 2020

\section{References}

1. Maiti, K, Malagoli, M. C., Magnano, E., Dallmeyer, A. \& Carbone, C. Electronic band structure of Gd: a consistent description. Phys. Rev. Lett. 86, 2846-2849 (2001).

2. Hughes, I. D. et al. Lanthanide contraction and magnetism in the heavy rare earth elements. Nature 446, 650-653 (2007).

3. Oroszlány, L., Deák, A., Simon, E., Khmelevskyi, S. \& Szunyogh, L. Magnetism of gadolinium: a first-principles perspective. Phys. Rev. Lett. 115, 096402 (2015).

4. Mendive-Tapia, E. \& Staunton, J. B. Theory of magnetic ordering in the heavy rare earths: ab initio electronic origin of pair- and four-spin interactions. Phys. Rev. Lett. 118, 197202 (2017).

5. Aruna, I., Mehta, B. R., Malhotra, L. K. \& Shivaprasad, S. M. Stability and hydrogenation of 'bare' gadolinium nanoparticles. Adv. Funct. Mater. 15, 131-137 (2005)

6. Kuei, P. Y. \& Hu, C. C. Gadolinium oxide high-k gate dielectrics prepared by anodic oxidation. Appl. Surf. Sci. 254, 5487-5491 (2008).

7. Chaudhari, P., Cuomo, J. J. \& Gambino, R. J. Amorphous metallic films for magneto-optic applications. Appl. Phys. Lett. 22, 337-339 (1973).

8. Kim, K-J. et al. Fast domain wall motion in the vicinity of the angular momentum compensation temperature of ferrimagnets. Nat. Mater. 16, 1187-1192 (2017).

9. Hirata, Y. et al. Correlation between compensation temperatures of magnetization and angular momentum in GdFeCo ferrimagnets. Phys. Rev. B 97, 220403 (2018).

10. Caretta, L. et al. Fast current-driven domain walls and small skyrmions in a compensated ferrimagnet. Nat. Nanotechnol. 13, 1154-1160 (2018).

11. Hirata, Y. et al. Vanishing skyrmion Hall effect at the angular momentum compensation temperature of a ferrimagnet. Nat. Nanotechnol. 14, 232-236 (2019).

12. Kim, D.-H. et al. Low magnetic damping of ferrimagnetic GdFeCo alloys. Phys. Rev. Lett. 122, 127203 (2019).

13. Cohen, S., Shamir, N., Mintz, M. H., Jacob, I. \& Zalkind, S. The interaction of $\mathrm{O}_{2}$ with the surface of polycrystalline gadolinium at the temperature range 300-670 K. Surf. Sci. 605, 1589-1594 (2011).

14. Gilbert, D. A. et al. Structural and magnetic depth profiles of magneto-ionic heterostructures beyond the interface limit. Nat. Commun. 7, 12264 (2016).

15. Bauer, U., Emori, S. \& Beach, G. S. D. Electric field control of domain wall propagation in Pt/Co/GdO x films. Appl. Phys. Lett. 100, 192408 (2012).

16. Bauer, $U$. et al. Magneto-ionic control of interfacial magnetism. Nat. Mater. 14, 174-181 (2015).

17. Bauer, U., Emori, S. \& Beach, G. S. D. Voltage-controlled domain wall traps in ferromagnetic nanowires. Nat. Nanotechnol. 8, 411-416 (2013).

18. Huang, M. et al. Three-terminal resistive switch based on metal/metal oxide redox reactions. Sci. Rep. 7, 7452 (2017).

19. Bi, C., Xu, M., Almasi, H., Rosales, M. \& Wang, W. Metal based nonvolatile fieldeffect transistors. Adv. Funct. Mater. 26, 3490-3495 (2016).

20. Lim, J. S. et al. Ultrafast collective oxygen-vacancy flow in Ca-doped $\mathrm{BiFeO}_{3}$ NPG Asia Mater. 10, 943-955 (2018).

21. Liu, L. et al. Oxygen vacancies: the origin of n-type conductivity in $\mathrm{ZnO}$. Phys. Rev. B 93, 235305 (2016).

22. Wang, Z. Q. et al. Synaptic learning and memory functions achieved using oxygen ion migration/diffusion in an amorphous $\operatorname{lnGaZnO}$ memristor. Adv. Funct. Mater. 22, 2759-2765 (2012).

23. Hiraoka, T. \& Suzuki, M. Magnetoresistance effect in single crystals of Gd. J. Phys. Soc. Jpn. 31, 1361-1365 (1971).

24. Raquet, B., Viret, M., Sondergard, E., Cespedes, O. \& Mamy, R. Electron-magnon scattering and magnetic resistivity in 3d ferromagnets. Phys. Rev. B 66, 024433 (2002). 
25. Mihai, A. P., Attané, J. P., Marty, A., Warin, P. \& Samson, Y. Electron-magnon diffusion and magnetization reversal detection in FePt thin films. Phys. Rev. $B$ 77, 060401 (2008)

26. Xiao, J. Q., Jiang, J. S. \& Chien, C. L. Giant magnetoresistance in nonmultilayer magnetic systems. Phys. Rev. Lett. 68, 3749-3752 (1992).

27. Gerber, A. et al. Magnetoresistance of granular ferromagnets. Phys. Rev. B $\mathbf{5 5}$ 6446-6452 (1997).
28. Honda, S., Okada, T., Nawate, M. \& Tokumoto, M. Tunneling giant magnetoresistance in heterogeneous $\mathrm{Fe}_{-} \mathrm{SiO}_{2}$ granular films. Phys. Rev. B $\mathbf{5 6}$ 14566-14573 (1997)

29. Sheng, P., Abeles, B. \& Arie, Y. Hopping conductivity in granular metals. Phys. Rev. Lett. 31, 44-47 (1973).

30. Helman, J. S. \& Abeles, B. Tunneling of spin-polarized electrons and magnetoresistance in granular Ni films. Phys. Rev. Lett. 37, 1429-1432 (1976). 